J3eA, Journal sur l'enseignement des sciences et technologies de l'information et des systèmes, Volume 2, Hors-Série 1, 4 (2003)

DOI : http://dx.doi.org/10.1051/bib-j3ea:2003504

(C) EDP Sciences, 2003

\title{
Optimisation et théorie de la commande robuste
}

D. Arzelier, J. Bernussou, D. Henrion et D. Peaucelle

\section{LAAS-CNRS}

7 av. du colonel Roche

F-31077 Toulouse cedex 04, France

(arzelier, bernussou, peaucelle, henrion) dlaas.fr 


\title{
Optimisation et théorie de la commande robuste
}

\author{
D. Arzelier, J. Bernussou, D. Henrion et D. Peaucelle \\ LAAS-CNRS, 07, Avenue du colonel Roche, 31077 Toulouse cedex 04 \\ (arzelier, bernusou, peaucelle,henrion)@laas.fr
}

Mots-clefs : théorie de la commande robuste, optimisation globale, programmation semidéfinie positive

\section{Introduction}

Le paradigme central en théorie de la commande robuste repose sur la structure de commande à contre-réaction et sur les modèles linéaires à temps invariant (LTI) des systèmes dynamiques (cf. figure 1). La première utilise l'information des sorties mesurées y pour élaborer un signal de commande $u$. Les modèles LTI quant à eux sont des objets mathématiques bien caractérisés et simples à utiliser. Cette simplicité n'est toutefois pas sans conséquence sur la précision relative de ce modèle et sur sa représentativité du système physique réel. Il est donc nécessaire d'adjoindre au modèle LTI un modèle mathématique de l'incertitude représentant l'écart entre la réalité physique et le modèle mathématique simplifé. Le fait que le modèle LTI est incertain est alors formalisé par la dépendance du modèle vis-à-vis d'un opérateur d'incertitude $\Delta$.

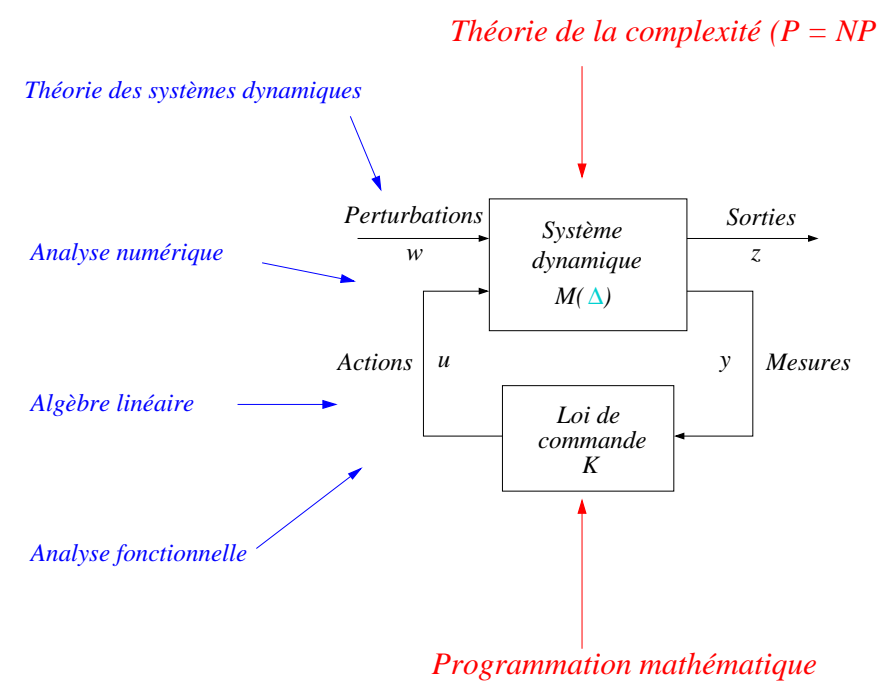

FIG. 1 - Paradigme standard de la commande robuste

L'objectif principal de la structure est d'assurer la stabilité de la boucle fermée ainsi qu'un certain degré de performance, mais aussi de réduire la sensibilité de la structure de commande en présence des variations paramétriques et d'éventuelles perturbations affectant le modèle du système à commander. Ces dernières sont regroupées dans le vecteur d'entrées exogènes $w$ dont il s'agit de minimiser l'influence sur les signaux controlés importants et pertinents regroupés dans le vecteur $z$. Le paradigme standard de la commande robuste ainsi défini permet de regrouper sous un même formalisme l'ensemble des problèmes rencontrés en théorie de la commande et déclinés suivant les trois axes de la modélisation, de l'analyse et de la synthèse.

Traditionnellement, les méthodes permettant d'aborder les problèmes de modélisation, de conception et d'analyse des systèmes de commande élaborés dans ce cadre font appel aux champs 
mathématiques traditionnels, analyse fonctionnelle, analyse numérique... Le développement des méthodes de point intérieur et le développement de la programmation semidéfinie positive [41] ont sensiblement modifié ce contexte. En effet, les problèmes pré-cités ne trouvent qu'à de rares exceptions des solutions analytiques littérales complètes (résolution d'équations de Riccati par exemple). Dans la majorité des cas, les problèmes d'analyse et de synthèse robustes se reformulent comme des problèmes de programmation mathématique possédant de multiples extrema locaux. Parmi ceux-là, seuls quelques cas bien identifiés conduisent à des problèmes convexes (utilisant le formalisme des inégalités matricielles linéaires, LMI) [14]. La différence entre résultats forts et résultats faibles se manifeste alors au travers de la complexité calculatoire (temps polynomial ou non des algorithmes de résolution associés) et au travers de la démonstrabilité globale (solutions locales ou globales des conditions posées, convexité ou non convexité des problèmes d'optimisation associés).

Les récents développements en programmation semidéfinie positive et en optimisation globale ont montré que les échanges entre les communautés de la théorie de la commande et de l'optimisation sont souvent à l'origine d'avancées significatives dans l'une ou l'autre des communautés [51]. Notre but est donc de rappeler quelques avancées dans le domaine pour ensuite définir un certain nombre d'enjeux actuels liés à l'application des méthodes et résultats de l'optimisation en théorie de la commande.

\section{Quelques problèmes d'optimisation en commande robuste}

\subsection{Modélisation incertaine}

Seuls les problèmes d'analyse et de synthèse seront abordés ici . Afin de ne pas surcharger cet article, nous nous contentons de citer quelques problèmes caractéristiques et d'y associer les références que nous jugeons les plus pertinentes. La modélisation et l'obtention du modèle incertain nécessitent un travail complexe qui conditionne en majeure partie le choix de la méthode de résolution des problèmes d'analyse et de synthèse ainsi que son efficacité. Etant donné qu'il s'agit essentiellement d'optimiser le compromis entre la complexité du modèle produit et sa simplicité d'utilisation, cette étape implique dans la majorité des cas la résolution de problèmes d'optimisation complexes et de grande taille. En particulier, les problèmes de réduction de modèles et d'identification robuste conduisent tout naturellement à la définition de problèmes d'optimisation non convexes pour lesquels des méthodes de résolution adéquates doivent être développées.

Dans le premier cas, la transformation fractionnaire linéaire de Redheffer [56] a permis de donner un cadre formel unifié et puissant mais pour lequel l'obtention systématique de la réalisation minimale du modèle incertain LFT reste ouvert [9]. La réduction d'ordre de ces modèles LFT, cruciale pour permettre leur utilisation efficace, suppose de résoudre un problème de minimisation du rang d'un produit de matrices symétriques définies positives sur un ensemble convexe [8], [9], [33].

De tels problèmes d'optimisation apparaissent également en identification robuste pour l'identification paramétrique $\mathcal{H}_{\infty}$ dans le domaine fréquentiel [39], [32] mais également pour la validation de modèle [27].

\subsection{Synthèse robuste}

Le modèle incertain du système étant défini, il s'agit de calculer une loi de commande compatible avec la structure en contre-réaction de la figure 1 et qui confère au système le plus insensible possible aux incertitudes et perturbations pouvant l'affecter tout en permettant d'atteindre un 
niveau de performances satisfaisant. Cela implique le choix d'une structure de commande par la définition de spécifications fonctionnelles et de classe mais également le choix d'une méthode de synthèse. Etant donnée la diversité des approches et des cadres de travail possibles, ce texte est volontairement limité à deux problèmes classiques de la théorie de la commande robuste: la synthèse de loi de commande par retour de sortie statique (incluant tous les problèmes de synthèse avec complexité réduite) et la synthèse multiobjectif.

\subsubsection{La synthèse par retour de sortie statique}

Soit le modèle d'état linéaire temps-invariant (LTI) :

$$
\begin{aligned}
& \dot{x}(t)=A x(t)+B u(t) \\
& y=C x
\end{aligned} \quad \text { où } x \in \mathbb{R}^{n}, y \in \mathbb{R}^{r} \text { et } u \in \mathbb{R}^{m} .
$$

Il s'agit de résoudre le problème suivant :

\section{Problème 1 :}

Déterminer $K \in \mathbb{R}^{r \times m}$ telle que $A+B K C$ soit stable i.e. le spectre de la matrice en boucle fermée $A+B K C$ est inclu dans le demi-plan complexe gauche.

$$
\Lambda(A+B K C) \subset \mathbb{C}^{-}
$$

Ce problème qui n'est pas stricto sensu un problème de commande robuste n'en reste pas moins d'une importance primordiale en théorie de la commande et particulièrement dans les cas pratiques. En effet, dans la majorité des cas, les problèmes pratiques de commande doivent être résolus sous l'hypothèse restrictive d'information partielle. Le système de commande ne dispose pas de la connaissance complète de l'état du système afin d'élaborer la loi de commande puisque seules les mesures fournies par les capteurs (en nombre limité) lui sont accessibles. Il est intéressant de remarquer également que de très nombreux problèmes de synthèse (synthèse par retour de sortie d'ordre réduit, synthèse de correcteur PID) peuvent être reformulés ainsi.

Si l'on sait que le problème 1 est décidable [1], sa complexité théorique n'a jamais été clairement établie [13]. Depuis les premiers travaux sur le sujet, de nombreuses approches différentes existent [50]. Celle fondée sur l'utilisation du critère de stabilité de Routh-Hurwitz associé aux méthodes d'élimination des quantificateurs de Tarski-Seidenberg issues de la théorie de la décision [1] permet de reformuler le problème initial comme celui de la recherche d'une solution à un système d'inégalités polynomiales multivariables dont les indéterminées sont les éléments de la matrice $K$. Un angle d'approche différent consiste à utiliser la théorie de Lyapunov afin de dériver des conditions nécessaires et suffisantes d'existence. Ces conditions consistent en la recherche de solutions à des inégalités matricielles non linéaires (bilinéaires) et/ou linéaires avec une condition de rang. Il est donc nécessaire d'identifier un élément dans un ensemble non convexe.

\section{Théorème 1 :}

Le problème de retour de sortie statique a une solution ssi

- $\exists K \in \mathbb{R}^{r \times m}$ et $\exists P \in \mathcal{S}_{n}^{+}$solutions de :

$$
(A+B K C)^{\prime} P+P(A+B K C)<\mathbf{0}
$$


- [36], $\exists(X, Y) \in \mathcal{S}_{n}^{+} \times \mathcal{S}_{n}^{+}$solutions de :

$$
\begin{aligned}
& B^{\perp}\left(A X+X A^{\prime}\right) B^{\perp^{\prime}}<\mathbf{0} \\
& C^{\prime \perp}\left(A^{\prime} Y+Y A\right) C^{\prime \perp^{\prime}}<\mathbf{0}
\end{aligned} \quad X=Y^{-1} \text { ou rang }\left[\begin{array}{cc}
X & \mathbf{1} \\
\mathbf{1} & Y
\end{array}\right]=n
$$

- [47], $\exists(P, X, Z) \in \mathcal{S}_{n} \times \mathcal{S}_{r} \times \mathcal{S}_{m}$ et $\exists Y \in \mathbb{R}^{r \times m}$ solutions de :

$$
\begin{aligned}
& Z>\mathbf{0} \quad P>\mathbf{0} \quad X \leq Y Z^{-1} Y^{\prime} \\
& {\left[\begin{array}{ll}
\mathbf{1} & \mathbf{0} \\
A & B
\end{array}\right]^{\prime}\left[\begin{array}{ll}
\mathbf{0} & P \\
P & \mathbf{0}
\end{array}\right]\left[\begin{array}{cc}
\mathbf{1} & \mathbf{0} \\
A & B
\end{array}\right]<\left[\begin{array}{cc}
C & D \\
\mathbf{0} & \mathbf{1}
\end{array}\right]^{\prime}\left[\begin{array}{cc}
X & Y \\
Y^{\prime} & Z
\end{array}\right]\left[\begin{array}{cc}
C & D \\
\mathbf{0} & \mathbf{1}
\end{array}\right]}
\end{aligned}
$$

Les trois formulations précédentes font clairement apparaitre que le problème de retour de sortie statique se reformule indifféremment comme un problème de recherche de solutions d'une BMI (inégalité matricielle bilinéaire), de solutions de LMI (inégalités matricielles linéaires) couplées par une égalité/inégalité non linéaire ou une contrainte de rang.

\subsubsection{La synthèse mixte $\mathcal{H}_{2} / \mathcal{H}_{\infty}$}

Depuis la fin des années 80 , les théories de la synthèse $\mathcal{H}_{2}$ et de la synthèse $\mathcal{H}_{\infty}$ sont théoriquement bien établies. Ce sont donc principalement les applications de ce type de synthèse dans l'espace d'état à des cas pratiques de plus en plus complexes qui se sont développées ces dernières années. La confrontation de ces théories très élégantes mais en partie fermées a conduit à faire deux constats complémentaires. D'une part, il est bien connu depuis les travaux présentés dans [18], que le régulateur LQG peut présenter des marges de robustesse vis-à-vis d'incertitudes paramétriques, dramatiquement et arbitrairement faibles. D'autre part, les nombreuses applications récentes des compensateurs $\mathcal{H}_{\infty}$ à des problèmes pratiques ont également montré qu'il est relativement difficile d'obtenir avec ce type de correcteurs un niveau satisfaisant de performances mesurées par exemple par la norme $\mathcal{H}_{2}$. Cela provient en partie du fait que la synthèse $\mathcal{H}_{\infty}$ est essentiellement fondée sur l'analyse de la performance dans le pire des cas alors que la norme $\mathcal{H}_{2}$ reflète une performance moyenne.

Face à ces deux constats, l'idée d'associer ces deux types de performance a éclos à la fin des années 80 en prenant la forme de la définition du problème mixte $\mathcal{H}_{2} / \mathcal{H}_{\infty}$ dont le schéma standard est représenté figure 2.

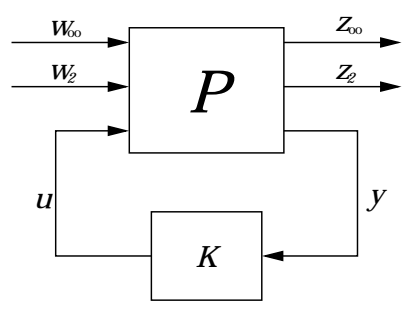

FIG. 2 - Modélisation générale du problème $\mathcal{H}_{2} / \mathcal{H}_{\infty}$

avec :

$$
P(s):=\left[\begin{array}{c|ccc}
A & B_{\infty} & B_{2} & B \\
\hline C_{\infty} & D_{\infty} & \mathbf{0} & D_{\infty u} \\
C_{2} & \mathbf{0} & \mathbf{0} & D_{2 u} \\
C & D_{y \infty} & \mathbf{0} & \mathbf{0}
\end{array}\right]
$$


Problème 2 : problème $\mathcal{H}_{2} / \mathcal{H}_{\infty}$

Etant donné un niveau de performance $\mathcal{H}_{\infty}$ admissible $\gamma$, déterminer un retour de sortie statique $u(t)=K y(t)$, admissible, $K \in \mathcal{K}_{\text {rss }}$, tel que :

$$
\begin{array}{ll}
\inf _{K \in \mathcal{K}_{r s s}} & \left\|T_{z_{2} w_{2}}\right\|_{2} \\
\text { sous } & \left\|T_{z_{\infty} w_{\infty}}\right\| \|_{\infty}<\gamma
\end{array}
$$

L'objectif est, à travers la résolution de ces différents problèmes, de résoudre un problème d'optimisation des performances nominales de la boucle fermée sous une contrainte de stabilité robuste et d'intégrer ainsi l'exigence des performances optimisées et de robustesse en un seul correcteur. Cela est illustré avec l'exemple très simple suivant emprunté dans [38] :

$$
\begin{aligned}
& A=\left[\begin{array}{cc}
0 & 1 \\
-1 & 0
\end{array}\right] \quad B=\left[\begin{array}{l}
0 \\
1
\end{array}\right] \quad C=\left[\begin{array}{ll}
0 & 1
\end{array}\right] \\
& C_{2}=\left[\begin{array}{ll}
1 & 0 \\
0 & 0
\end{array}\right] \quad B_{2}=\mathbf{1}_{2} \quad D_{2 u}=\left[\begin{array}{l}
0 \\
1
\end{array}\right] \\
& C_{\infty}=\left[\begin{array}{ll}
0 & 1
\end{array}\right] \quad B_{\infty}=\left[\begin{array}{l}
1 \\
0
\end{array}\right] \quad D_{\infty u}=0 \\
& D_{\infty}=0 \quad D_{y \infty}=0
\end{aligned}
$$

Cela permet d'écrire le problème d'optimisation équivalent:

$$
\begin{array}{ll}
\min _{k} & J(k)=\sqrt{-\frac{2+3 k^{2}}{2 k}} \\
& k<0 \\
& f(k)=\frac{2}{\sqrt{k^{2}\left(4-k^{2}\right)}} \leq \gamma
\end{array}
$$

Ce dernier peut être résolu aisément graphiquement comme illustré en figure 3 .

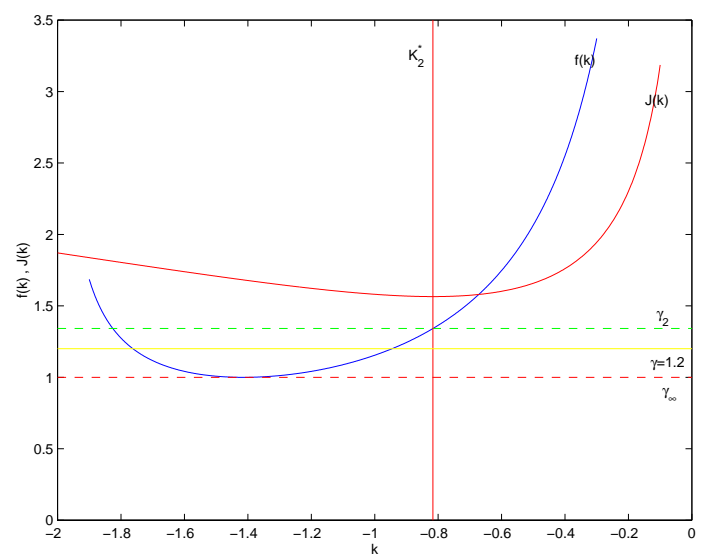

FIG. 3 - Résolution graphique du problème mixte 
Dans le cas général, en notant $P_{b f}$ le modèle en boucle fermée:

$$
\begin{aligned}
P_{b f}^{r s s}(s) & :=\left[\begin{array}{c|cc}
A_{b f} & B_{\infty b f} & B_{2 b f} \\
\hline C_{\infty b f} & D_{\infty b f} & \mathbf{0} \\
C_{2 b f} & D_{2 \infty b f} & \mathbf{0}
\end{array}\right] \\
& :=\left[\begin{array}{c|cc}
A+B K C & B_{\infty}+B K D_{y \infty} & B_{2} \\
\hline C_{\infty}+D_{\infty u} K C & D_{\infty}+D_{\infty u} K D_{y \infty} & \mathbf{0} \\
C_{2}+D_{2 u} K C & D_{2 u} K D_{y \infty} & \mathbf{0}
\end{array}\right]
\end{aligned}
$$

on obtient le résultat suivant:

\section{Lemme 1}

Les conditions suivantes sont équivalentes:

1- Il existe un gain de retour statique de sortie $K^{*}$ solution du problème de synthèse mixte 2.

2- Il existe $\left(X_{2}, X_{\infty}\right) \in \mathcal{S}_{n}^{+} \times \mathcal{S}_{n}^{+}$et un gain de retour de sortie statique $K \in \mathcal{K}_{\text {rss }}$ solutions du problème d'optimisation:

$$
\begin{gathered}
\min _{\substack{X_{2}, X_{\infty}, K \\
\text { sous }}} \operatorname{trace}\left(B_{2 b f}^{\prime} X_{2} B_{2 b f}\right) \\
A_{b f}^{\prime} X_{2}+X_{2} A_{b f}+C_{2 b f}^{\prime} C_{2 b f}<\mathbf{0} \\
{\left[\begin{array}{cc}
A_{b f}^{\prime} X_{\infty}+X_{\infty} A_{b f}+C_{\infty b f}^{\prime} C_{\infty b f} & X_{\infty} B_{\infty b f}+C_{\infty b f}^{\prime} D_{\infty b f} \\
\star & D_{\infty b f}^{\prime} D_{\infty b f}-\gamma^{2} \mathbf{1}
\end{array}\right]<\mathbf{0}}
\end{gathered}
$$

La solution du problème mixte passe donc par la résolution d'un problème BMI. Quoique les théories $\mathcal{H}_{2}$ et $\mathcal{H}_{\infty}$ individuelles soient parfaitement maîtrisées, le problème mixte $\mathcal{H}_{2} / \mathcal{H}_{\infty}$ reste jusqu'à maintenant un problème très largement ouvert. En effet, mis à part quelques résultats particuliers analytiques, nous ne disposons pas de la solution littérale du problème ni de la solution numérique que pourrait apporter le cadre de travail LMI. En effet, quelque soit le type de loi de commande envisagée (retour d'état ou de sortie statique ou dynamique) les différentes approches sont sous-optimales.

\subsection{Analyse robuste}

La problématique générale de l'analyse robuste peut s'énoncer comme suit. Etant donnée une modélisation incertaine et une loi de commande associée, il s'agit d'établir si cette loi de commande garantit la stabilité et un certain niveau de performance de la boucle de contreréaction pour toute réalisation du modèle dans son ensemble d'incertitude. A de très rares exceptions, les problèmes d'analyse de la stabilité et de la performance robustes sont résolus complètement. Dans la majorité des cas, il est nécessaire de poser un problème d'optimisation complexe non convexe et possédant peu de propriétés fortes. Nous allons illustrer cela sur un problème simple qui est toujours ouvert: l'analyse de stabilité robuste d'un polytope de matrices. 
Soit le modèle autonome incertain linéaire temps invariant:

$$
\begin{gathered}
\dot{x}(t)=A x(t) \quad \sum_{i=1}^{N} \lambda_{i} A^{[N]} \lambda_{i}>0 \sum_{i=1}^{N} \lambda_{i}=1 \\
A \in \mathcal{A}=\operatorname{co}\left\{A^{[1]}, \cdots, A^{[N]}\right\}
\end{gathered}
$$

La matrice dynamique $A$ n'est pas précisément connue mais est supposée appartenir à un polytope de matrices $\mathcal{A}$. Toute réalisation $A$ de la matrice dynamique peut s'exprimer comme une combinaison linéaire convexe des $N$ matrices sommets $A^{[1]}, \cdots, A^{[N]}$.

\section{Problème 3 :}

Le polytope de matrices $\mathcal{A}$ est-il stable?

$$
\Lambda(A) \subset \mathbb{C}^{-} \quad \forall A \in \mathcal{A}
$$

Coxson et DeMarco dans [17] ont montré que ce problème est $\mathcal{N} \mathcal{P}$-difficile. Après qu'il a été montré à l'aide de contre-exemples que la conjecture des sommets proposée par Bialas [12] était fausse, des méthodes géométriques [16] et combinatoires [55] ont permis de proposer des conditions nécessaires et suffisantes qui ne sont toutefois pas opérationnelles ou difficilement testables numériquement. En combinant la théorie de Lyapunov et la théorie des jeux, Chen [15] a proposé une interprétation nouvelle du problème 3 . Définissant

$$
\mathcal{P}=\left\{P: P>\mathbf{0}, \lambda_{\max }(P)=1\right\}
$$

il est possible de reformuler le test de stabilité robuste comme celui de la valeur d'un jeu à deux joueurs et somme nulle.

\section{Théorème 2 :}

$\mathcal{A}$ est stable ssi:

$$
J=\max _{A \in \mathcal{A}} \min _{P \in \mathcal{P}} \lambda_{\max }\left(A^{\prime} P+P A\right)=\max _{A \in \mathcal{A}} \phi(A)<0
$$

Ce dernier est un problème non trivial de théorie des jeux $(\phi(A)$ généralement non différentiable et non concave) pour lequel il est difficile de tester la condition du point-selle.

$$
\exists\left(A^{*}, P^{*}\right) \in \mathcal{A} \times \mathcal{P} \mid \lambda_{\max }\left(A^{\prime} P^{*}+P^{*} A\right) \leq \lambda_{\max }\left(A^{*^{\prime}} P^{*}+P^{*} A^{*}\right) \leq \lambda_{\max }\left(A^{*^{\prime}} P+P A^{*}\right)
$$

\section{4 $\mathrm{SDP}+\mathrm{LMI}=\mathrm{CR} ?$ ou $\mathrm{NLP}+\mathrm{BMI}=\mathrm{TC} ?$}

Depuis les mutations introduites en théorie de la commande par le paradigme standard et par le formalisme LMI, la somme de nouveaux résultats en commande robuste ramenant le calcul de la solution à celui de la résolution d'un problème de programmation semidéfinie positive a pu laisser penser qu'il suffisait de trouver la bonne formulation LMI du problème initial pour ensuite le résoudre numériquement avec un algorithme de SDP adéquat. Il a fallu rapidement en rabattre et plutôt constater que la plupart des problèmes de l'Automatique dite moderne conduisaient dans la majorité des cas à poser des problèmes d'optimisation globale (l'hypothèse de convexité n'etant pas vérifiée) complexes et pour la plupart des cas réalistes d'applications, de grande taille. Pour tous les formalismes actuellement utilisés en analyse et synthèse robustes allant de la théorie du $\mu$ (analyse et synthèse) au cadre de travail défini par les contraintes intégrales quadratiques, en passant par la théorie de la séparation des graphes, il est nécessaire de disposer d'une théorie de l'optimisation adéquate ainsi que des outils numériques efficaces associés. 


\subsection{Différentes classes des problèmes d'optimisation}

Comme nous venons de le voir, la théorie de l'optimisation est au coeur des développements actuels et futurs en théorie de la commande robuste. Il est toutefois remarquable de constater qu'il est possible de présenter l'ensemble de ces problèmes dans un cadre unifié. Ainsi, quatre grandes familles peuvent être identifiées.

- Le problème BMI :

$$
\begin{array}{ll}
\min & \sum_{i=1}^{n} c_{i} x_{i} \\
\text { sous } & \\
& A+\sum_{i=1}^{n} B_{i} x_{i}+\sum_{i=1}^{n} \sum_{j=i}^{n} C_{i, j} x_{i} x_{j} \prec \mathbf{0}
\end{array}
$$

où $A, B_{i}, C_{i, j}$ sont des matrices symétriques données.

- Le problème LMI avec contrainte de rang:

$$
\begin{array}{ll}
\min & \sum_{i=1}^{n} c_{i} x_{i} \\
\text { sous } & A+\sum_{i=1}^{n} B_{i} x_{i}<\mathbf{0} \\
& \operatorname{rang}\left(C+\sum_{i=1}^{n} D_{i} x_{i}\right) \leq N
\end{array}
$$

où $A, B_{i}, C, D_{i}$ sont des matrices symétriques données.

- Le problème de minimisation concave:

$$
\begin{array}{ll}
\min & f(x) \\
\text { sous } & A+\sum_{i=1}^{n} B_{i} x_{i}<\mathbf{0}
\end{array}
$$

où $A, B_{i}$ sont des matrices symétriques données et $f$ une fonction concave.

- Le problème de complémentarité conique:

$$
\begin{array}{ll}
\min & \operatorname{Trace}(Z F) \\
\text { sous } & (F, Z) \in \mathcal{S}
\end{array}
$$

où $F$ et $Z$ sont des matrices appartenant au cône $\mathcal{S}$ des matrices symétriques semidéfinies positives.

Sauf à exploiter la structure proposée par des cas particulier (monotonie du problème de complémentarité conique [19] ou de la minimisation de rang [40]), il n'existe pas encore de résultats théoriques forts ni algorithmes réalistes et efficaces sur de tels problèmes.

\section{Quelques approches usuelles}

Les différentes approches afin de traiter de tels problèmes en analyse et synthèse robustes peuvent être classées en deux grandes familles. La première regroupe les méthodes fondées plus ou moins directement sur l'utilisation de relaxations convexes du problème original alors que la seconde est caractérisée par l'emploi de techniques issues de l'optimisation globale. 


\subsection{Théorie de Lyapunov et relaxation convexe}

Historiquement, les premières approches ont fait appel à des résultats directement issus de la théorie de la commande (théorie de Lyapunov, lemme d'élimination...) [14], [46] afin de donner des conditions suffisantes d'existence auxquelles des bornes supérieures sont associées. Nous donnons des exemples simples dans chaque cas.

Nous reprenons l'exemple de la section 2.3 qui concerne l'analyse robuste en stabilité d'un polytope de matrices. La relaxation proposée consiste à permutter le min et le max dans le problème de théorie des jeux associé et de définir une borne supérieure $J_{\text {quad }}$ sur la valeur $J$ du jeu initial :

$$
J=\max _{A \in \mathcal{A}} \min _{P \in \mathcal{P}} \lambda_{\max }\left(A^{\prime} P+P A\right)<J_{\text {quad }}=\min _{P \in \mathcal{P}} \max _{A \in \mathcal{A}} \lambda_{\max }\left(A^{\prime} P+P A\right)
$$

Cette relaxation bien connue en théorie de la commande robuste [6] revient à tester la stabilité quadratique du polytope de matrices qui est une condition suffisante pour la stabilité robuste.

\section{Théorème 3 :}

Si

$$
\exists P>\mathbf{0} \mid A_{i}^{\prime} P+P A_{i}<\mathbf{0} \quad \forall i=1, \cdots, N
$$

alors $\dot{x}=A x$ est stable $\forall A \in \mathcal{A}=c o\left\{A^{[1]}, \cdots, A^{[N]}\right\}$.

Une interprétation complémentaire de celle proposée par la théorie des jeux permet de montrer que la matrice $P$ n'est rien d'autre qu'une variable de Lagrange issue d'une relaxation Lagrangienne du problème de stabilité robuste initial [19].

Le pessimisme introduit par la relaxation précédente peut être important et il est possible de le réduire en dérivant une relaxation plus fine à l'aide du lemme d'elimination ou lemme de Finsler.

\section{Lemme 2 :}

Soient $\mathcal{L} \in \mathcal{S}^{n}$ et $\mathcal{R} \in \mathbb{R}^{m \times n}$ avec $\operatorname{rang}(\mathcal{R})<n:$

$$
\mathcal{R}^{\perp^{\prime}} \mathcal{L} \mathcal{R}^{\perp}<\mathbf{0} \quad \Leftrightarrow \quad \exists H \in \mathbb{R}^{n \times m} \mid \mathcal{L}+H \mathcal{R}+\mathcal{R}^{\prime} H^{\prime}<\mathbf{0}
$$

Il est ainsi possible de déduire une nouvelle condition suffisante de stabilité robuste dont on peut montrer qu'elle est toujours meilleure que celle fondée sur la notion de stabilité quadratique [45].

\section{Théorème 4 :}

Si $\exists N$ matrices $P_{i}>\mathbf{0}, F$ et $G$

$$
\left[\begin{array}{cc}
\mathbf{0} & P_{i} \\
P_{i} & \mathbf{0}
\end{array}\right]+\left[\begin{array}{c}
A_{i}^{\prime} \\
-\mathbf{1}
\end{array}\right]\left[\begin{array}{ll}
F^{\prime} & G^{\prime}
\end{array}\right]+\left[\begin{array}{c}
F \\
G
\end{array}\right]\left[\begin{array}{cc}
A_{i} & \mathbf{1}
\end{array}\right]<\mathbf{0} \quad \forall i=1, \cdots, N
$$

alors $\dot{x}=A x$ est stable $\forall A \in \mathcal{A}=\operatorname{co}\left\{A^{[1]}, \cdots, A^{[N]}\right\}$.

De nouveau, les variables matricielles intervenant dans ce test de stabilité robuste, les $N$ matrices de Lyapunov et les matrices $F$ et $G$ peuvent être réinterprétées comme des variables issues de relaxations Lagrangiennes du problème initial. Il est possible ainsi de multiplier les exemples de méthodes d'analyse et de synthèse robustes qui sont intrinsèquement fondées sur des relaxations Lagrangiennes cachées du problème initial. 


\subsection{Les relaxations Lagrangiennes et hiérarchiques}

Comme nous venons de le voir, les problèmes de programmation semidéfinie positive obtenus en théorie de la commande robuste sont intrinsèquement issus de relaxations Lagrangiennes des problèmes d'optimisation (souvent quadratiques) originaux [19], [42]. Nous en rappelons le principe général. Soit le problème de programmation mathématique:

$$
\begin{array}{ll}
\inf _{x \in \mathcal{X}} & f(x) \\
\text { sous } & h_{i}(x) \leq 0 \quad i=1, \cdots, m
\end{array}
$$

En définissant le Lagrangien par la fonction $\mathcal{L}(x, \lambda)=f(x)+\sum_{i=1}^{m} \lambda_{i} h_{i}(x) \quad \lambda_{i} \geq 0$, il est possible d'écrire le problème dual associé à (11) :

$$
\sup _{\lambda \in \mathbb{R}^{+m}} \inf _{x \in \mathcal{X}} \mathcal{L}(x, \lambda)=\sup _{\lambda \in \mathbb{R}^{m+}} \theta(\lambda)
$$

La fonction duale $\theta(\lambda)$ est concave et semi-continue supérieurement. Résoudre le dual du dual revient à résoudre une relaxation convexe de (11). Pour une introduction plus complète aux relaxations convexes et à la dualité Lagrangienne, on peut se reporter aux références [42] et [34].

La $\mathcal{S}$-procédure essentielle en théorie de la commande [52], [53] et à l'origine de très nombreux résultats [14] est l'exemple paradigmatique d'un outil mathématique connu et largement utilisé de manière indépendante par les deux communautés de l'optimisation [49] et de la commande [54]. Ainsi, l'interprétation de la $\mathcal{S}$-procédure comme une application particulière de la relaxation de Lagrange des problèmes quadratiques $\left(h_{i}\right.$ sont des fonctions quadratiques) [35], [19] est relativement peu connue dans la communauté Automatique.

Un autre exemple est constitué par la relaxation de rang très usuelle en optimisation combinatoire (max-cut) [26], [28] et dont on peut montrer qu'elle constitue une relaxation duale pour le problème de stabilité robuste abordé par la théorie de Lyapunov dans la section précédente [19], [29].

La grande faiblesse des relaxations Lagrangienne est qu'elles peuvent être très pessimistes (le saut de dualité peut être arbitrairement grand). L'évaluation du degré de pessimisme peut constituer en soi un problème très difficile à résoudre [51]. Pour une certaine classe de problèmes, il est toutefois possible de proposer des résultats plus forts. Soient $g_{i}(x), i=0,1, \cdots, m$ des fonctions polynomiales à plusieurs indéterminées. Nous définissons le problème d'optimisation sur l'ensemble des polynômes positifs :

\section{Problème 4:}

$$
\begin{array}{ll}
\min _{x \in \mathbb{R}^{n}} & g_{0}(x) \\
\text { sous } & g_{k}(x) \geq 0 \quad k=1, \cdots, m
\end{array}
$$

Une hiérarchie de relaxations LMI successives a été proposée indépendemment dans [51] à partir de l'exploitation des propriétés de la dualité conique, dans [44] par la décomposition des polynômes en somme de carrés dans le cadre de la géométrie algébrique (propriétés des ensembles semi-algébriques) et de manière duale dans [37] par la théorie des moments. Cette hiérarchie de relaxations convexes de moins en moins pessimistes quand l'ordre augmente possède la propriété de garantir asymptotiquement la convergence de la hiérarchie vers l'optimum global du problème (13). Cela se paie bien évidemment par l'augmentation du nombre de variables et du nombre de contraintes au passage d'une relaxation donnée à une relaxation d'ordre supérieure. De nombreux exemples en optimisation globale, optimisation combinatoire et théorie de la commande sont présentés dans la référence [30]. 


\subsection{Les relaxations heuristiques}

Un autre type de relaxation très souvent utilisé en théorie de la commande consiste à utiliser un schéma de relaxations SDP successives fondé sur une heuristique. Ce type d'approche est essentiellement utilisé pour la résolution de problèmes du type (7) et revient à une linéarisation du problème bilinéaire initial. Cette linéarisation prend usuellement deux formes distinctes.

La première est connue comme une extension de la méthode du gradient contraint au problème (10) ou au problème (9). Schématiquement, étant donnée une fonction non linéaire $f$ de variables matricielles $X$ et $Y$ et un ensemble réalisable défini par une contrainte LMI $\mathcal{L}(X, Y)$ :

\section{Problème 5 :}

$$
\begin{array}{ll}
\min _{X, Y} & f(X, Y) \\
\text { sous } & \mathcal{L}(X, Y)<0
\end{array}
$$

La méthode consiste à résoudre itérativement le problème LMI linéarisé suivant:

$$
\begin{array}{ll}
\min _{X, Y} & \nabla_{\left(X_{k}, Y_{k}\right)} f(X, Y) \\
\text { sous } & \mathcal{L}(X, Y)<0
\end{array}
$$

A chaque étape $k$, un problème LMI doit être résolu fournissant une solution optimale qui est utilisée pour le calcul du gradient à l'étape suivante. Cette méthode a été particulièrement utilisée dans les cas particuliers $f(X, Y)=\operatorname{trace}(X Y)[19]$ et $f(X, Y, Z)=\operatorname{trace}\left(X-Y Z^{-1} Y\right)[2]$. Il est à noter que l'on dispose d'une solution réalisable simplement calculable à chaque étape et en particulier pour initialiser le processus itératif. On a également la garantie de la décroissance monotone du critère. Il n'est toutefois pas rare d'observer un phénomène de plateau ne conduisant pas même à un optimum local pour lequel aucune garantie n'est fournie. Ce problème peut être contourné en utilisant des méthodes hybrides incluant des informations au second ordre (hessien) [21]. Les tests d'arrêt pour un tel algorithme sont également délicat à définir.

Une alternative heuristique permet d'aborder la classe des problèmes d'optimisation ne rentrant pas dans le cadre SDP et définit comme suit:

\section{Problème 6 :}

$$
\begin{array}{ll}
\min _{(X, Y)} & f(X, Y, X Y) \\
\text { sous } & \mathcal{L}(X, Y, X Y)<0
\end{array}
$$

où $f$ et $\mathcal{L}$ sont des fonctions bilinéaires en les variables $X$ et $Y$ ne contenant pas de termes quadratiques homogènes en les variables $X$ et $Y$.

Le principe de la méthode s'inspire des méthodes classiques de descente coordonnée où l'on identifie deux sous-ensembles de variables distinctes $X$ et $Y$. Cela permet de décomposer le problème d'optimisation conjoint en $(X, Y)$ en une série d'optimisations croisées consécutives en chacune des variables $X$ et $Y$. Le processus d'optimisation est un processus itératif dont chaque itération $k$ est divisée en deux demi-itérations :

$$
\begin{array}{llll}
\min _{X} & f\left(X, Y_{k}, X Y_{k}\right) & \min _{Y} & f\left(X_{k}, Y, X_{k} Y\right) \\
\text { sous } & \mathcal{L}\left(X, Y_{k}, X Y_{k}\right)<0 & \text { sous } & \mathcal{L}\left(X_{k}, Y, X_{k} Y\right)<0
\end{array}
$$

Cette approche est très populaire en théorie de la commande où la séparation des variables est souvent naturelle (variables d'analyse et variables de synthèse) [43], [24], [4]. D'une grande 
facilité de mise en oeuvre, l'évolution du critère dans ce type d'algorithmes itératifs possède la propriété de décroissance monotone alors que les tests d'arrêt peuvent être aisément définis. En revanche, l'initialisation peut s'avérer périlleuse et la convergence vers un optimum local n'est pas garantie.

\subsection{L'optimisation globale}

Cette famille d'approches repose sur l'application directe au problème (7) de méthodes d'optimisation globale. Deux grandes tendances se regroupent autour des méthodes pures de branch and bound et les techniques fondées sur la décomposition généralisée de Benders.

Dans le premier cas, l'espace de recherche est découpé de manière plus ou moins heuristique et des bornes inférieures et supérieures sont calculées sur les sous-domaines ainsi définis. Le processus global doit permettre de faire converger les bornes supérieures et inférieures vers l'optimum global. On peut trouver quelques applications de ces techniques en théorie de la commande [5], [25], [2]. Les techniques de branch and bound induisent pour des problèmes de grande taille une charge calculatoire importante voire rédhibitoire pour certaines applications.

Dans le deuxième cas, la décomposition généralisée de Benders [23] a été utilisée pour développer un algorithme primal relaxé-dual. L'algorithme GOP présenté dans [22] a été appliqué avec succès sur des problèmes particuliers d'analyse paramétrique et polynomiale de la marge de stabilité robuste [48]. Il a été également étendu au problème de synthèse par retour de sortie statique où les coefficients de la matrice $K$ sont supposés être bornés en amplitude [11].

Même si le problème initial est effectivement dans la majorité des cas un problème d'optimisation globale, les dernières techniques présentées ne donnent pas entièrement satisfaction de par leur lourdeur de mise en oeuvre et leur complexité de réglage pour un non spécialiste tel que peut l'être un automaticien désireux d'analyser la robustesse d'une boucle d'asservissement industrielle ou de régler une loi de commande efficace.

\section{Quelques enjeux de l'optimisation en théorie de la commande}

$\mathrm{Au}$ delà des résultats passés et actuels liant les deux communautés, il apparaît que la communauté de la théorie de la commande doit encore mieux profiter des développements théoriques et numériques en programmation mathématique.

$\mathrm{Au}$ plan théorique, le concept de dualité Lagrangienne (notamment en programmation semidéfinie positive) a permis des interprétations pertinentes et prometteuses pour l'analyse du pessimisme des conditions développées en théorie de la commande robuste [10]. Pour autant, ces résultats sont préliminaires et semblent pouvoir être étendus dans d'autres domaines en Automatique. L'exemple des relaxations hiérarchiques obtenues pour les problèmes d'optimisation sur les polynômes positifs nous font penser que l'on sera certainement amené à aller au delà de la dualité Lagrangienne.

Sur le plan algorithmique, il est évident que les solutions proposées actuellement pour résoudre le problème (7) ne sont pas complètement satisfaisantes tant du point de vue des garanties théoriques offertes (convergence, aspect global de la solution) que des potentialités purement numériques (grande taille, réalisabilité exacte des solutions incontournable en $\mathrm{Au}$ tomatique, conditionnement numérique...). Une approche prometteuse consiste à étendre les techniques d'optimisation différentielle au cône SDP pour la recherche de solutions locales avec garantie de convergence (méthode du Lagrangien augmenté [20]). L'extension des méthodes de barrière [41] au cas non convexe est également actuellement un sujet d'intérêt majeur au sein de 
la communauté. Enfin, l'évaluation des méthodes non différentiables (plans de coupe, méthodes des liasses) [42], [31] sur des exemples significatifs et pertinents tant en taille qu'en complexité doit également être menée.

Ces éléments permettent de considérer qu'un domaine d'études original et situé à la frontière des deux champs scientifiques est encore largement ouvert.

\section{Références}

[1] B.D.O. Anderson, N.K. Bose, E.I. Jury, "Output feedback stabilization and related problems-solutions via decision methods", IEEE Transactions on Automatic control, Vol. 20, No. 1, pp. 53-66, 1975.

[2] P. Apkarian, H.D. Tuan, "Robust control via concave minimization: local and global algorithm", IEEE Transactions on Automatic Control, Vol. 45, No. 2, pp. 299-305, Février 2000 .

[3] D. Arzelier, J. Bernussou, D. Peaucelle, "Fonctions de lyapunov dépendant des paramètres pour l'analyse et la synthèse robuste", chapître 6 dans Conception de commandes robustes, sous la direction de Jacques Bernussou et Alain Oustaloup, Traité IC2, Hermes, 2002.

[4] D. Arzelier, D. Peaucelle, "An iterative method for $H_{2} / H_{\infty}$ synthesis via static output feedback", Proceedings of the 41st IEEE Conference on Decision and Control, Las Vegas, Nevada, 2002.

[5] V. Balkrishnan, S. Boyd, S. Balemi, "Branch and bound algorithm for computing the minimum stability degree of parameter-dependent linear systems", dans C.T. Leondes éditeur, Control and dynamic systems, Vol. 53, Academic Press, 1992.

[6] B.R. Barmish, "Necessary and sufficient conditions for quadratic stabilizability of an uncertain linear system", JOTA, Vol. 46, No. 4, 1985.

[7] B.R. Barmish, M. Fu, S. Saleh, "Stability of a polytope of matrices", IEEE Transactions on Automatic Control, Vol. 33, pp. 569-572, 1988.

[8] C. Beck, "Minimality for uncertain systems and IQCs", Proceedings of the 33rd Conference on Decision and Control, Lake Buena Vista, FL, Décembre 1994.

[9] C. Beck, R. D'Andrea, "Computational study and comparisons of LFT reducibility methods", Proceedings of the American Control Conference, Philadelphia, Pennsylvania, Juin 1998.

[10] A. Ben-Tal, A. Nemirovskii, Lectures on modern convex optimization analysis, algorithms and engineering applications, SIAM Studies in Applied Mathematics, 2001.

[11] E. Beran, L. Vandenberghe, S. Boyd, "A global BMI algorithm based on the generalized Benders decomposition", Proceedings of the European Control Conference, Bruxelles, Belgique, 1997.

[12] S. Bialas, "A necessary and sufficient condition for stability of interval matrices", Int. J. of Control, Vol. 37, No. 5, pp. 1103-1104, 1983.

[13] V. Blondel, E.D. Sontag, M. Vidyasagar, J.C. Willems, Open problems in mathematical systems and control theory, Springer Verlag, 1998. 
[14] S. Boyd, L. El Ghaoui, E. Feron, V. Balakrishnan, Linear Matrix Inequalities in System and Control Theory, SIAM Studies in Applied Mathematics, Philadelphia, 1994.

[15] W.H. Chen, "On relationship between quadratic and robust stability of uncertain systems", Int. J. of Robust and Nonlinear Control, Vol. 9, pp. 51-58, 1999.

[16] J.D. Cobb, C.L. DeMarco, "The minimal dimension of stable faces required to guarantee stability of a matrix polytope", IEEE Transactions on Automatic Control, Vol. 34, No. 9, Septembre 1989.

[17] G.E. Coxson, C.L. DeMarco, "Testing robust stability of general matrix polytope is an $\mathcal{N P}$-hard computation", Proceedings of the Allerton Conference, 1991.

[18] J. Doyle, "Guaranteed margins for LQG regulators", IEEE Transactions on Automatic Control, Vol 23, No.4, pp. 756-757, 1978.

[19] L. El Ghaoui, S. Niculescu, Advances in linear matrix inequality methods in control, SIAM advances in design and control, 2000.

[20] B. Farès, P. Apkarian, D. Noll, "An augmented Lagrangian method for a class of LMIconstrained problems in robust control theory", International Journal of Control, Vol. 74, No. 4, pp. 348-360, 2001.

[21] B. Fares, D. Noll, P. Apkarian, "Robust control via sequential semidefinite programming", SIAM Journal on Control and Optimization, Vol. 40, No. 6, pp. 1791-1820, 2002.

[22] C.A. Floudas, V. Visweswaran, "A global optimization algorithm for certain classes of nonconvex NLPs, I Theory", Computers and Chimical Engineering, Vol. 14, No. 12, pp. 1397-1417, 1990.

[23] A.M. Geoffrion, "Generalized Benders decomposition", JOTA, Vol. 10, No. 4, 1972.

[24] J.C. Geromel, J. Bernussou, M.C. de Oliveira, " $H_{2}$ norm optimization with constrained dynamic output feedback controllers: Decentralized and reliable control", IEEE Transactions on Automatic Control, Vol. 44, No. 7, pp. 1449-1454, Juillet 1999.

[25] K. Goh, M.G. Safonov, G. P. Papavaissilopoulos, "Global optimization for the biaffine inequality problem", Journal of global optimization, Vol. 7, pp. 365-380, 1995.

[26] M.X. Goemans, D.P. Williamson, ".878-approximation algorithms for MAX-CUT and MAX-2SAT", Proceedings of the 26th Annual Symposium on Theory of Computing, Montréal, pp. 422-431, 1994.

[27] S. Gugercin, A.C. Antoulas, "On consistency and model validation for systems with parametric uncertainty", Proceedings of IFAC Symposium on System Identification, Santa Barbara, CA, Juin 2000.

[28] D. Henrion, S. Tarbouriech, D. Arzelier, "LMI approximations for the radius of the intersection of ellipsoids: Survey", JOTA, Vol. 108, No. 1, pp. 1-28, Janvier 2001.

[29] D. Henrion, G. Meinsma, "Rank one LMIs and Lyapunov's inequality", IEEE Transactions on Automatic Control, Vol. 46, No. 8, pp. 1285-1288, Août 2001.

[30] D. Henrion, J. B. Lasserre, "GloptiPoly: Global Optimization over Polynomials with Matlab and SeDuMi", Proceedings of the IEEE Conference on Decision and Control, Las Vegas, Nevada, Décembre 2002. 
[31] C. Helmberg, F. Rendl, "A spectral bundle method for semidefinite programming", SIAM Journal on Optimization, 2001.

[32] A.J. Helmicki, C.A. Jacobson, C.N. Nett, "Control oriented system identification: a worstcase/deterministic approach in $\mathcal{H}_{\infty}$ ", IEEE Transactions on Automatic control, Vol. 36, pp. 1163-1176, 1991.

[33] A. Hiret, C. Valentin-Charbonnel, G. Duc, J.P. Bonnet, "Different multidimensional reduction algorithms for the LFT model of a missile", 2nd IMACS International multiconference CESA, Hammamet, Tunisie, 1998.

[34] J.B. Hiriart-Urruty, C. Lemaréchal, Convex Analysis and Minimization Algorithms I,II, Springer Verlag, 1991.

[35] J.B. Hiriart-Urruty, M. Torki, "Permanently going back and forth between the "quadratic world" and the "convexity world" in optimization", Applied Mathematics and Optimization

[36] T. Iwasaki, R.E. Skelton, J.C. Geromel, "Linear quadratic suboptimal control with static output feedback", Systems and Control Letters, Vol. 23, No. 6, pp. 421-430, 1994.

[37] J.B. Lasserre, "Global optimization with polynomials and the problem of moments", SIAM J. On Optim., Vol. 11, No. 3, pp. 796-817, 2001.

[38] W. Levine, M. Athans, "On the determination of the optimal constant output feedback gains for linear multivariable systems", IEEE Transactions on Automatic control, Vol. 15, pp. 44-48, 1970.

[39] E. van der Meché, O. Bosgra, "A convex relaxation approach to real rational frequency domain identification", Proceedings of the 41st Conference on Decision and Control, Las Vegas, Nevada, Décembre 2002.

[40] M. Mesbahi, "On the rank minimization problem and its control application", Systems and Control Letters, Vol. 33, pp. 31-36, 1998.

[41] Y. Nesterov, A. Nemirovskii, "Interior-point polynomial algorithms in convex programming", SIAM Studies in applied mathematics, 1994.

[42] F. Oustry, "Duality, dissipativity and LMIs", lecture at the graduate school, université catholique de Louvain-la-Neuve, Novembre 2000.

[43] A. Packard, J. Doyle, "The complex structured singular value"”, Automatica, Vol. 21, No. 1, pp. 71-109, 1993.

[44] P.A. Parillo, "Higher order semidefinite relaxations for quadratic programming", Proceedings of the 39th IEEE Conference on Decision and Control, Sidney, Australie, 2000.

[45] D. Peaucelle, D. Arzelier, O. Bachelier, J. Bernussou, "A new robust D-stability condition for real convex polytopic uncertainty", Systems and Control Letters, Vol. 40, pp. 21-30, 2000 .

[46] D. Peaucelle, D. Arzelier, "Robust performance analysis with LMI-based methods for real parametric uncertainty via parameter-dependent Lyapunov functions", IEEE Transactions on Automatic Control, Vol.46, No. 4, pp. 624-630, Avril 2001.

[47] D. Peaucelle, D. Arzelier, "Ellipsoidal sets for static output feedback", Proceedings of the Automatic Control 15th IFAC World Congress, Barcelona, Espagne, 2002. 
[48] P. Psarris, C. A. Floudas, "Robust stability analysis of systems with real parametric uncertainty: A global optimization approach", International Journal of Robust and Nonlinear Control, Vol. 5, pp. 699-717, 1995.

[49] N. Shor, "Quadratic optimization problems", Soviet J. Circuits Systems Sci., Vol. 25, pp. $1-11,1987$.

[50] V.L. Syrmos, C.T. Abdallah, P. Dorato, K. Grigoriadis, "Static output feedback-A survey", Automatica, Vol. 33, No. 2, pp. 125-137, 1997.

[51] H. Wolkowicz, R. Saigal, L. Vandenberghe, Handbook of semidefinite programming, Kluwer's international series, 2000.

[52] V.A. Yakubovich, "S-procedure in nonlinear control theory", Vestnik Leningrad Univ., Vol. 1, pp. 62-77, 1971.

[53] V.A. Yakubovich, "Minimization of quadratic functionals under quadratic constraints and the necessity of a frequency condition in the quadratic criterion for absolute stability of nonlinear control systems", Soviet Math. Dokl., Vol. 14, No. 2, pp. 593-597, 1973.

[54] V.A. Yakubovich, "Nonconvex optimization problem: The infinite-horizon linear-quadratic control problem with quadratic constraints", Systems Control Letters, Vol. 19, pp. 13-22, 1992.

[55] R. K. Yedavalli, "A necessary and sufficient extreme point solution for checking robust stability of polytopes of matrices", Proceedings of the American Control Conference, Chicago, Illinois, Juin 2000.

[56] K. Zhou, J. Doyle, K. Glover, Robust and Optimal Control, Prentice Hall, New Jersey, 1996. 